\title{
Sirtuins as therapeutic targets of ALS
}

\author{
Cell Research (2013) 23:1073-1074. doi:10.1038/cr.2013.94; published online 16 July 2013
}

Sirtuins have received a lot of
attention in biological functions as-
sociated with metabolism, survival
development, and most recently, neu-
rodegeneration. The versatile role of
sirtuins can be readily redirected for
drug discovery studies for novel treat-
ment in amyotrophic lateral sclerosis (ALS), as presented in this highlight, by sirtuin-mediated ketogenic responses influencing mitochondrial function.

Modulation of sirtuin activity has been shown to impact the course of several aggregate-forming neurodegenerative disorders including Alzheimer's disease (AD), Parkinson's disease, Huntington's disease, amyotrophic lateral sclerosis (ALS) and spinal and bulbar muscular atrophy. Sirtuin proteins possess either mono-ribosyltransferase or deacetylase activity. Sirtuins have been linked with caloric restriction (CR) and aging by modulating energy metabolism, genomic stability and stress resistance. For example, our laboratory was the first to demonstrate that promotion of the $\mathrm{NAD}^{+}$-dependent SIRT1-mediated deacetylation, may be a mechanism by which CR influences AD-type amyloid neuropathology [1].

Mammals contain seven sirtuins (SIRT1-7) that occupy different subcellular compartments such as the nucleus (SIRT1, 2, 6, 7), cytoplasm (SIRT1 and 2 ) and the mitochondria (SIRT3, 4 and 5). Class I sirtuins (SIRT1-3) possess robust deacetylase activity in the presence of $\mathrm{NAD}^{+}$, whereas Class II sirtuins (SIRT4) have ADP-ribosyltransferase activity. Class III sirtuins (SIRT5) possess $\mathrm{NAD}^{+}$-dependent demalonylase and desuccinylase activities in addition to deacetylase activity. The class IV sirtuins (SIRT6 and 7) are deacetylase enzymes that may be weaker and more substrate-specific than the class I deacetylases in vitro, and SIRT6 has also been shown to possess ADPribosyltransferase activity. Mitochondrial sirtuins (SIRT3, 4 and 5) play an important role in cellular ROS defense and mitochondrial energy metabolism. Moreover, SIRT3 has been shown to be regulated by PGC-1 $\alpha$, which can promote mitochondrial biogenesis and is associated with ROS suppression and neuroprotection $[2,3]$.

Although the effect of sirtuin proteins on aging has been challenged [4], sirtuin enzymes are still potential therapeutic targets in a variety of human diseases including cancer, diabetes, inflammatory disorders and neurodegenerative diseases, including ALS. ALS is a progressive neurodegenerative disease that affects nerve cells in the brain and the spinal cord. Mutations in SOD1, TDP43, FUS, UBPLN2 and C9ORF72 have been identified in familial ALS cases, although the majority ( $\sim 90 \%)$ of ALS cases are sporadic. ALS patients have been documented to have impaired energy balance. This defect in energy metabolism has been linked to lower activities of mitochondrial electron transport chain enzymes in ALS spinal cord [5], and improving mitochondrial function may represent a therapeutic approach for ALS. Our recent findings suggest that medium chain triglyceride (MCT) treatment could lead to an increased blood ketone level, protect motor neuron loss and attenuate ALS-type motor impairment, possibly through promoting oxygen consumption rate [6].
Altered sirtuin levels have been observed in both SOD1-G93A mouse models [7] and patient tissue [8], suggesting that the sirtuin family may be a disease target both in ALS mouse models and humans. Although treatment with dietary resveratrol, a SIRT1 activator, was not sufficient to exert any impact on disease outcomes, intraperitoneal injection of resveratrol led to a significant improvement in both symptoms and survival in the SOD1G93A mouse model of ALS [7]. These different findings may be due to the short half-life of resveratrol as well as different routes of administration. Recent studies identified SIRT3 as a relevant player in ALS pathogenesis using a cell-based model. SIRT3 was shown to protect against mitochondrial fragmentation and neuronal cell death induced by SOD1-G93A overexpression [9]. Moreover, PGC-1 $\alpha$, which has been shown to promote SIRT3 [2], showed the same effect as SIRT3 in this model. Interestingly, SIRT3 was also shown to regulate ketone body production by deacetylating mitochondrial 3-hydroxy-3-methylglutaryl CoA synthase 2 (HMGCS2) [10]. This evidence may be consistent with our recent pilot study showing the elevation of mitochondrial sirtuin expression in MCT-treated primary motor neuron cultures and in the spinal cord of SOD1G93A mice following MCT treatment (Pasinetti lab, unpublished data). Based on the above information, we hypothesize that MCT might regulate mitochondrial activity and cell survival through sirtuin-mediated responses. The mechanisms that we are proposing for targeting sirtuins in ALS pathogenesis 


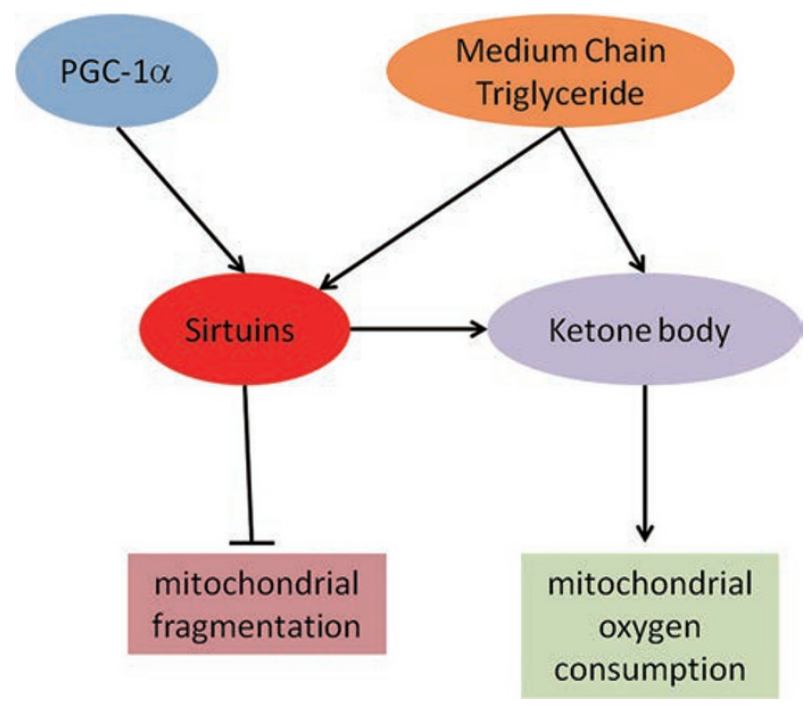

Figure 1 Scheme of targeting sirtuins in ALS pathogenesis. Sirtuins inhibit mitochondrial fragmentation and promote ketone body level. Medium chain triglyceride increases both sirtuin expression and ketone body level in SOD1-G93A mouse model of ALS.

are diagrammed in Figure 1. Additional research confirming the effect of pharmacologic intervention in animal models of ALS will be important in evaluating whether sirtuin modulatory compounds should enter the human clinical trial pipeline.

\section{Giulio Maria Pasinetti ${ }^{1,2}$,} Amanda E Bilski ${ }^{1}$, Wei Zhao ${ }^{1,2}$
${ }^{1}$ Department of Neurology, Mount Sinai School of Medicine, 1468 Madison Avenue, Annenberg Building, Room 20-02, New York, NY 10029, USA; ${ }^{2}$ GRECC, James $J$ Peters Veterans Affairs Medical Center, Bronx, NY, USA

Correspondence: Giulio Maria Pasinetti

Tel: $+1-212-241-7938$ or $+1-212-241-5563$

Fax: +1-212-876-9042

E-mail: Giulio.Pasinetti@mssm.edu

\section{References}

1 Qin W, Yang T, Ho L, et al. J Biol Chem 2006; 281:21745-21754.

2 Giralt A, Hondares E, Villena JA, et al. J Biol Chem 2011; 286:16958-16866.

3 Zhao W, Varghese M, Yemul S, et al. Mol Neurodegener 2011; 6:51.

4 Viswanathan M, Guarente L. Nature 2011; 477:E1-E2.

5 Swerdlow RH, Parks JK, Cassarino DS, et al. Exp Neurol 1998; 153:135-142.

6 Zhao W, Varghese M, Vempati P, et al. PLoS One 2012; 7:e49191.

7 Han S, Choi JR, Soon Shin K, et al. Brain Res 2012; 1483:112-117.

8 Korner S, Boselt S, Thau N, et al. Neurodegener Dis 2013; 11:141-152.

9 Song W, Song Y, Kincaid B, et al. Neurobiol Dis 2013; 51:72-81.

10 Shimazu T, Hirschey MD, Hua L, et al. Cell Metab 2010; 12:654-661. 